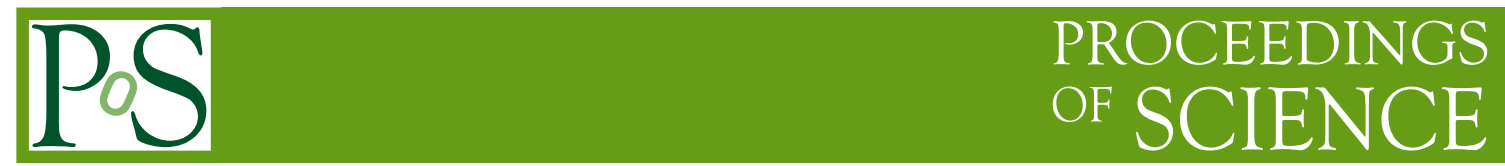

\title{
Alternative dark matter candidates: axions and ALPs
}

\section{Javier Redondo*}

Departamento de Física Teórica, Universidad de Zaragoza, 50009 Zaragoza

Max-Planck-Institut für Physik, 80805 München, Germany

E-mail: jredondo@unizar.es

The QCD axion and particles with similar properties (axion-like particles or ALPs) are excellent candidates to be the cold dark matter of the Universe. They are extremely different to the standard WIMP, however. In this talk I presented and highlighted the mostly shocking aspects of axion dark matter, the prospects for its discovery and the recent blossoming of experimental techniques to search for these elusive particles, which more than ever are now receiving a lot of interest.

Neutrino Oscillation Workshop (NOW2018)

9 - 16 September, 2018

Rosa Marina (Ostuni, Brindisi, Italy)

${ }^{*}$ Speaker. 


\section{Axions and ALPs}

The QCD axion is a hypothetical meson-like $0^{-}$particle predicted by the Peccei-Quinn mechanism $[1,2,3,4]$ to solve the strong CP problem dynamically using the very dynamics of QCD (quantum chromo-dynamics). The axion field adds up to the only flavour-neutral $\mathrm{CP}$ violating parameter allowed in the standard model (SM), the $\theta$-angle,

$$
\mathscr{L} \ni \frac{1}{2}\left(\partial_{\mu} a\right)\left(\partial^{\mu} a\right)-\frac{\alpha_{s}}{8 \pi} \widetilde{G}_{a \mu \nu} G_{a}^{\mu \nu}\left(\theta+\frac{a}{f_{a}}\right)
$$

because the vacuum energy density caused by QCD instantons, $V_{q c d}$, is sensitive essentially only to the parameter multiplying the gluonic Chern-Simmons term $\widetilde{G}_{a \mu \nu} G_{a}^{\mu v}$ and has a minimum at $\theta-\langle a\rangle / f_{a}=0$. This potential energy drives the axion VEV to cancel any value of $\theta$, which is very welcome in the SM since it is the combination of two unrelated and in principle large terms, $\theta=\theta_{q c d}+\operatorname{argDet}\left(\mathrm{M}_{\mathrm{q}}\right)$. The dynamical relaxation allows to explain comfortably the absence of a neutron electric-dipole-moment, $d_{n}<3 \times 10^{-13} \mathrm{fm}$ [5], because the axion VEV also appears in the prediction $d_{n}=0.0024(10)\left(\theta+\langle a\rangle / f_{a}\right) \mathrm{fm}$, cancelling the CP violation dynamically. In the very simplest realisation of the PQ mechanism, there is only one parameter, a new energy scale $f_{a}$ required to couple $a$ to $\widetilde{G}_{a \mu v} G_{a}^{\mu \nu}$. This corresponds to a new energy scale in physics to be identified. The axion particle acquires a mass $m_{a}^{2}=\partial_{a}^{2} V_{q c d}=\chi / f_{a}^{2}$ where the QCD topological susceptibility is $\chi=(75.5 \mathrm{MeV})^{4}[6,7]$.

The cancellation mechanism requires the axion theory to posses a shift symmetry ${ }^{1} a \rightarrow a+\alpha f_{a}$ (called PQ symmetry) to be only violated by the QCD term in Eq. 1.1. This constraints the lowenergy effective theory of any axion model to have a few extra types of couplings to SM fields,

$$
\mathscr{L}_{i}=\sum_{f} C_{a f f^{\prime}}\left[\bar{f} \gamma^{\mu} \gamma_{5} f^{\prime}\right] \frac{\partial_{\mu} a}{f_{a}}-C_{a \gamma} \frac{\alpha}{8 \pi} \widetilde{F}^{\mu v} F_{\mu v} \frac{a}{f_{a}}-N \frac{\alpha_{s}}{8 \pi} \widetilde{G}_{a \mu \nu} G_{a}^{\mu v}\left(\theta+\frac{a}{v_{a}}\right),
$$

i.e. derivative couplings to fermions and an anomalous coupling to gauge bosons similar to the gluonic one. Axions are very easily realised as pseudo-Goldstone bosons but also appear in holomorphic theories with dynamical couplings like supersymmetry or string theory, where they appear model-independently $[8,9]$. Different axion models differ mostly in $f_{a}$ but also in their $\mathrm{O}(1)$ couplings $C_{a f f^{\prime}}, C_{a \gamma}$ and the so called domain wall number $N$ that counts how many values of $a / f_{a}=0,2 \pi, 4 \pi$ are physically equivalent $\mathrm{CP}$ conserving minima. The QCD axion must have $N \geq>1$ and then we can identify $f_{a}=v_{a} / N$. We can define an axion-like particle as having $N=0$ and getting a mass from some extra piece of physics breaking the shift symmetry. Classical QCD axion examples are the original PQWW axion based on a 2 Higgs doublet model (2HDM) with $f_{a} \sim v_{\mathrm{EW}}$ and soon ruled out, its high- $f_{a}$ versions DFSZ I and II, which only introduce an extra scalar with a large VEV $\sim f_{a}$ and the KSVZ model using not only a similar extra high-VEV scalar but also one or more quarks to generate the $\widetilde{G} G a$ term. All these models have flavour conserving interactions. A recent burst of axion model building has produced new models, in particular with non-trivial flavour interactions like the minimal-flavour-violating axion [10], the Flaxion/Axiflavon [11] and the astrophobic axions of [12], which feature small couplings to fermions that avoid

\footnotetext{
${ }^{1}$ This symmetry allows to redefine the standard $\theta$ to zero, which we do from now on. Indeed, one can now speak of the $a / f_{a}$ field playing the role of a dynamical version of $\theta$, or $\theta$ being promoted to a dynamical field.
} 
some astrophysical bounds, amongst others. All the couplings are inversely proportional to the new energy scale $f_{a}$, and so is the QCD axion mass but, what do we know already from it?

Axions and ALPs with relatively strong interactions $f_{a} \sim v_{\mathrm{EW}}$ could have been detected in colliders, beam dumps and reactor experiments but were not and are thus mostly excluded. Even larger values of $f_{a}$ up to $f_{a}>10^{7} \mathrm{GeV}$ are extremely toxic for stellar evolution as axion emission can contribute to the energy loss of stars more efficiently than neutrinos, a fact that is not observed and constrained in many well-known systems: the Sun, Helium-burning stars, white-dwarfs, redgiants and SN1987A to name the top. These axions and ALPs could also be produced thermally in the early Universe and constitute today hot or warm dark matter or dark radiation, observables quite constrained nowadays by precision cosmology data. This turned the interest into "invisible" axions, for which a huge value of $f_{a}>10^{7} \mathrm{GeV}$ - possibly related to intermediate scales, GUT scales or even the string-scale - is responsible for the feebleness of their interactions with the SM and their alpervading elusiveness. Such large values of $f_{a}$ imply the QCD axion having an extremely low mass, $m_{a} \lesssim 10 \mathrm{meV}$ or so (for ALPs one has much more freedom). The feebleness of their interactions and smallness of their mass determine the unique character of the axion as a dark matter candidate. The phenomenology of invisible axions is shared with other weaklyinteracting slim particles, which has fostered the coining of an acronym (WISPs) in opposition to the standard DM and BSM candidate, the WIMP. Up to now, WISPs have passed largely unnoticed - unintentionally honouring their name, something WIMPs are catching up with only now- but they are recently attracting more and more from the theory, pheno and, most importantly, from the experimental point of view at the low-energy frontier $[13,14,15,16]$.

Invisible axions and ALPs are excellent cold dark matter candidates, however. A first reason is that their cosmological zero modes (those with $k \sim H=\dot{R} / R$, is the Hubble expansion rate of the FRW scale factor $R$ ) are so weakly coupled to the SM plasma that they may never thermalise. The vacuum realignment mechanism explains how this is so. The idea is that the axion field is so weakly-interacting that it behaves as a completely decoupled field. Its initial conditions are set after a phase transition (hereby PQ phase transition) for example (the theory Eq. 1.2 is only valid at energies $\lesssim f_{a}$, one expects extra degrees of freedom above this scale) and then the field follows the decoupled equation of motion

$$
\ddot{a}-\frac{1}{R^{2}} \triangle a+H \dot{a}+\frac{\partial}{\partial a} V_{q c d}(a)=0,
$$

where $V_{q c d}$ is to be replaced in the case of an ALP. In the early Universe, the axion field redshifts as radiation from its initial condition, modes entering the horizon $(k>H)$ redshifting away and keeping a certain level of sub-horizon homogeneity. This picture continues until the term $\partial_{a} V_{q c d}(a) \sim m_{a}^{2} a$ becomes relevant and then it stars to perform damped oscillations around 0 at a frequency given by the axion mass $m_{a}$ and damping rate given by $H$. The energy density of the oscillating field damped by the Hubble expansion redshifts as $\rho \propto 1 / R^{3}$ exactly as cold dark matter. Since axions have very small masses this transition from effective-massless to DM happens relatively late in early cosmology: around temperatures $T \sim \mathrm{GeV}$ for $\mathrm{QCD}$ axions and even smaller $T$ for much lower mass ALPs. Note that some amount of CDM in axions and ALPs is generically unavoidable! as it follows from generic initial conditions and the feebleness of their interactions. Interestingly, the typical momenta of "axions" is of order $H$, extremely colder than the SM radiation 
bath. Indeed for radiation domination we have $H \propto T^{2} / M_{P}$ and thus the axion-to-SM "temperature" ratio is tiny, $\sim T / M_{p}$ with $M_{P}=1.2 \times 10^{19} \mathrm{GeV}$ the Planck mass. So axions, as any other bosonic WISP created by this realignment mechanism are indeed ultra cold DM. The axion/ALP yield depends unfortunately mostly upon $f_{a}$ and upon the initial conditions. Larger values of $f_{a}$ imply smaller $m_{a}$ and thus a later onset of the oscillations, i.e. less time with active damping until today and therefore more dark matter. The dependence with initial conditions is more bothering since it prevents to make robust and simple predictions. Two broad classes of initial conditions cover most cases: Post-inflationary and Pre-inflationary PQ phase transition. The latter is the simplest since inflation blows a sub-horizon patch where the axion field is largely homogeneous beyond the current observable Universe. Here, the initial value of the axion in this patch $a_{I}=\theta f_{a}$ is unpredictable, we have maximum uncertainty on the ICs! If $\theta_{I}$ is of the order of $\mathrm{O}(1)$, axions with $f_{a} \sim 10^{11} \mathrm{GeV}$ will give the totality of CDM but much larger values of $f_{a}$ can give the correct abundance if our patch of the Universe is anthropically biased to be $\theta_{I} \ll 1$, as suggested in [17]. A more predictive scenario is the Post-Inflationary one, where initial conditions for the axion or ALP field are set after inflation and cannot be coherent beyond the causal horizon $H^{-1}$. Unfortunately, a network of global cosmic strings (GCS) is generated by the Kibble mechanism [18] in the phase transition. These GCSs form loops that shrink and intersect when entering in the causal horizon resulting in a scaling solution where the string length per Hubble volume is $\xi \sim O(1)$. The energy per unit length stored in strings is huge $\mu \sim \pi f_{a}^{2} \log \left(f_{a} / H\right)$ and it is released into axions (axion waves) as the network relaxes loosing length. These axions constitute the subject of a long-standing controversy that has had a recent interesting chapter. We cannot simulate the dynamics of the GCSs because of the impossibility of resolving at the same time the string core $d_{c} \sim 1 / f_{a}$ and the size of Horizon $d_{H} 1 / H$. When axions become CDM the ratio $d_{H} / d_{c} \sim f_{a} / H \sim 10^{30}$ and our largest grids have reached only 8192 points per linear dimension, a mere factor of $10^{26}$ too short! Our simulations then effectively use values of $f_{a}$ much smaller than physical to be able to fit a couple of grid points inside of the core. Since the energy/length is only sensitive to the large log, one hopes for a solid extrapolation. A recent very nice study of the axion radiation from strings underlines that the extrapolation is still quite unsure [19], within current dynamical range the simulations predict the axion number spectrum to be IR dominated (at energies $\sim H$ ) but the axion energy spectrum to be UV dominated (at energies $\sim f_{a}$ ). Recently, a new technique has been proposed to at least put the same amount of energy/length in the strings, if not properly distributed of course, [20, 21]. Their results are consistent with the analysis of [19] despite the very different simulation and core energy. Taken at face-value the extrapolation implies the axion-DM mass to be $\sim 26 \mu \mathrm{eV}[21]$ and very few DM axions radiated from strings but [19] warns that this can be much larger if the axion spectrum hardens. Other recent estimates were $\sim 100 \mu \mathrm{eV}[22,23]$. If the axion mass is above this limit ( $f_{a}$ smaller than it should to give all DM) axions would be a subdominant component. If it is below, axions constitute too much DM and are excluded (in this scenario!). Thus we see that axions can give the correct DM abundance in a relatively broad range of parameters. Indeed the situation is even broader when we discuss two more aspects. First, all the above was outlined for $N=1$, case where the GCS network violently disappears when axions become CDM thanks to unstable domain walls built from $V_{q c d}$ that appear attached to the strings and drag them to oblivion. For $N>1$ there is more than $1 \mathrm{CP}$ conserving axion minimum and domain walls cannot shrink into a preferred vacuum, the GCS network continues to evolve in a sort of scaling regime with very 
energetic domain walls attached. The domain walls will end up dominating the expansion of the Universe, a fact in contradiction to observations. This pure $N>1$ post-inflationary PQ scenario is strongly excluded [24] but if PQ is explicitly broken the degeneracy between vacua is broken, domain-walls become unstable and the model is viable. Unfortunately the DM yield depends on the exact breaking and this is again model dependent although generally the axion DM mass in these scenarios tends to be larger than in the $N=1$ case. Very interesting models have been reviewed in [25]. The last important aspect is that these values of the axion DM mass hold for QCD axion in the case where the CDM transition takes place where the Universe is dominated by the SM radiation. Change this assumption and these values will change, see [26]. Finally, the above holds for the QCD axion. For other ALPs - whose mass for a given $f_{a}$ can be different - the CDM yield is $\Omega_{c d m} h^{2} \sim O(1) \sqrt{m_{a} / \mathrm{eV}}\left(f_{a} / 10^{12} \mathrm{GeV}\right)^{2}$ [27, 28] (The QCD axion case is a bit exception because its mass depends on temperature. Last high- $T$ lattice QCD calculations have made possible to make this calculation precisely, see [7]).

\section{Axion DM is different}

The very point of considering axions and ALPs as CDM candidates is that they behave slightly different than standard DM. Indeed they do it at many different levels: gravitationally and experimentally. Let us here briefly review them.

Axion CDM can be distinguished gravitationally from other forms of CDM. In the postinflationary scenario, the CDM distribution is expected to be inhomogeneous at scales $H^{-1}$ until it becomes CDM (time $t_{1}$ ) and then frozen. Regions of comoving size size $1 / R_{1} H\left(t_{1}\right)$ that are overdense $\mathrm{O}(1)$ with respect to others collapse gravitationally very early around matter-radiation equality forming $M \sim 10^{-12} M_{\odot}, R \sim 10^{12} \mathrm{~cm}$ axion miniclusters [29, 30, 31]. We have studied the distribution of the early seeds of these halos in numerical simulations including the GCS network for the first time in [32]. The ALP case has not been studied in detail but it is far more flexible in terms of minicluster properties [33]. Axion miniclusters have masses similar to primordial black holes recently constrained by the HSC microlensing survey of M31 [34]. Recent papers set very strong constraints on the fraction of DM in miniclusters [35, 36] mostly due to a relative aggressive choice of minicluster mass but this is now seemingly disfavoured by our results [32] and by recent critics raised against the microlensing analysis [37]. However, this is a potentially very efficient way of constraining the post-inflationary scenario and further surveys/analysis can produce a discovery or a total exclusion of the scenario. Another recently reviewed test is Femtolensing by the miniclusters [38], but relevant data is still missing. In this scenario, dilute axion stars [39] are expected to form in the cores of miniclusters [40,41] and possible trigger instabilities that convert some axions into axionic radiation [42] or into photons [43, 44, 45, 46] (although this possibility requires large couplings for QCD axions might be ok for other ALPs).

In the pre-inflationary scenario, the axion field is a good degree of freedom during inflation and their quantum fluctuations become stretched to horizon size where, if axion becomes a sizeable component of the CDM they will interact with the adiabatic temperature fluctuations originated by the inflaton. These uncorrelated axion CDM density fluctuations appear as isocurvature density anisotropies $[47,48]$ of the cosmic microwave background (CMB) and can be sought for in CMB data. The isocurvature power is $P_{\text {iso }} \sim H_{I}^{2} /\left(\pi^{2} f_{a}^{2} \theta_{I}^{2}\right)$ and it is constrained to be $P_{\text {iso }}<0.88 \times 10^{-10}$ 
by latest Planck data [49]. Unfortunately, the constraint depends on the Hubble rate of inflation, $H_{I}$, which is only constrained from above in slow-roll inflation to be $\lesssim 10^{14} \mathrm{GeV}$. For a sufficiently small $H_{I}$ axion CDM isocurvature anisotropies will pass unnoticed. The interesting twist to this story comes in the event of a near-future discovery of primordial gravitational waves (PGWs). The next generation CMB-polarisation experiments like LiteBird [50] can measure the power of PGWs generated during inflation, which is $\propto H_{I}^{2}$ and thus fix the main unknown in the axion isocurvature contribution. Since the improvement over the previous generation is modest (factor of $\sim 3$ in $H_{I}$ ) the potentially discoverable level of PGWs implies a huge amount of axion isocurvature fluctuations, and thus: 1) the exclusion of axion CDM in the pre-inflation scenario or 2) the imposition of axion CDM being a really subdominant component of the CDM or 3) that something in the axion model or the cosmology prevented standard quantum fluctuations of the axion during inflation, see for instance [51] and refs. therein. The funny 2014 episode of seeming detection of PGWs by Bicep2 triggered almost an immediate reaction from the community favouring the post-inflationary scenario [52].

The existence of axions or ALPs other potential gravitational hazards. A most remarkable one is the phenomenon of black-hole (BH) superradiance. If the Compton-wavelength of an axion, $1 / m_{a}$, is comparable to the Schwartzschild radius of a spinning blackhole, the phenomenon of superradiance kicks in allowing the exponential creation of an axion cloud around the $\mathrm{BH}$ that sucks the BH spin. The observation of maximally spinning BHs can therefore be used to constraint the existence of axions and ALPs [53]. The observation of quickly rotating stellar and supermassive BHs has been used to exclude the axion/ALP/WISP mass ranges $6 \times 10^{-13} \mathrm{eV}<m_{a}<2 \times 10^{-11} \mathrm{eV}$ and $10^{-17} \mathrm{eV}<m_{a}<7 \times 10^{-17} \mathrm{eV}$ respectively [53] although a much broader range is expected in the future $[53,54]$.

\section{The experimental quest}

Tiny oscillations of the axion field with amplitude $\theta_{0} \sim 3.6 \times 10^{-19}$ around the CP-conserving minimum would result in the local CDM density $\rho \sim 0.4 \mathrm{GeV} / \mathrm{cm}^{3}$ and can be attempted to be measured. The key of all the experimental techniques is employing a resonant device coupled to the axion oscillation frequency $\omega \sim m_{a}+O\left(m_{a} v^{2}\right)$ where the spectral width comes from the non-zero kinetic energy of galactic halo-axions, $v \sim 10^{-3}, \omega-m_{a} \sim 10^{-6}$, the axion oscillations have an equivalent quality factor of $10^{6}$. This implies a coherence length that can be quite macroscopic $\delta L=1 / \delta p \sim 20 m\left(10^{-5} \mathrm{eV} / m_{a}\right)$. The most direct way of testing the QCD nature of the DM would be to measure oscillations of the neutron or nuclear EDM. This is attempted by the CASPER experiment, which plans to be sensitive for sub neV axions after a few stages of R\&D [55]. The axion gradient couples to fermionic spins as a fictional oscillating B-field, which can be detected by a sensitive magnetometer (CASPER electric for nucleons or QUAX for electrons at much higher frequencies [56]). However most of axion DM searches (and in fact of the searches at all) are based on the axion-2-photon coupling. Axion DM can be searched by exciting a resonant microwave cavity (cavity "haloscope" of P.Sikivie [57]) tuned to the axion DM mass in a strong B-field. The ADMX experiment in Seattle leads the way and is taking extremely sensitive data as we speak around the sweet-spot for haloscopes $m_{a} \sim 3 \mu \mathrm{eV}$. A discovery could come at any moment [58] so it is worth staying in tune. The IBS center for axion and precision physics (CAPP) has been recently created 
with its main target of finding the axion by building different state of the art haloscopes around and above the ADMX search range (where axion DM is most natural). First results are expected soon. The HAYSTAC collaboration is a R\&D collaboration to test the improvements required for haloscopes to perform at higher frequencies (below quantum-limited detectors, superconducting cavities, high-volume resonators...). First results in the $m_{a} \sim 23 \mu \mathrm{eV}$ range [59] are already quite promising. Being the signal proportional to the cavity volume $\sim 1 / \mathrm{m}_{a}^{3}$ and the noise increasing with $m_{a}$ the haloscope concept requires modifications at higher energies. Multiple coupled cavities are being investigated by ADMX, CAPP and the RADES initiative [60], amongst others. A radical concept is to renounce completely to the resonant enhancement in favour of a much larger detector area as proposed by the dish antenna concept [61]. An intermediate concept is the dielectric haloscope attempted by the MADMAX collaboration $[62,63]$ to search for axion DM above the $40 \mu \mathrm{eV}$ mass range. This could be made to work at optical frequencies [64]. At energies below those attempted by the standard cavity technique, the DM radio or ABRACADABRA experiment attempts to detect the tiny dipolar B-field generated by solenoidal E-field oscillations induced in a strong B-field solenoid with potential sensitivity in the $\mathrm{neV}-\mu \mathrm{eV}$ range $[65,66,67]$. The list of new ideas grows thicker when we include experiments sensitive to ALPs with a stronger coupling to photons than the QCD axion.

Finally this little review would not really do without mentioning that axions and ALPs will lead to the phenomenon of light-shining-through walls sought with intense and exquisite IR lasers by the ALPS collaboration in DESY Hamburg [68], will be emitted copiously by the solar core and sought by axion helioscopes like CAST [69] at CERN or the future IAXO [70] at DESY and finally can lead to interesting long-range $\left(\sim 1 / m_{a}\right)$ forces to be scrutinised at an unprecedented level by the ARIADNE experiment [71]. Axion mediated flavoured transitions can be extremely sensitive, particularly in the $K^{+} \rightarrow \pi^{+} a$ decay which will be taken to the next level by the experiment NA62 [72].

In summary, the QCD axion and ALPs are very well motivated candidates to the CDM of the Universe but have a very different phenomenology and require very different experimental searches to WIMPs, the standard candidates. Ideas and interests are flourishing, some experiments taking data and others focus on R\&D and although a feeble spark of light can be said to have been lit, axions and ALPs will still remain as very interesting most likely remain as "alternatives" for the next few years.

\section{References}

[1] Peccei, R. and Quinn, H. R. Phys.Rev. D16, 1791-1797 (1977).

[2] Peccei, R. and Quinn, H. R. Phys.Rev.Lett. 38, 1440-1443 (1977).

[3] Weinberg, S. Phys.Rev.Lett. 40, 223-226 (1978).

[4] Wilczek, F. Phys.Rev.Lett. 40, 279-282 (1978).

[5] Baker, C., Doyle, D., Geltenbort, P., Green, K. et al. Phys.Rev.Lett. 97, 131801 (2006).

[6] Grilli di Cortona, G., Hardy, E., Pardo Vega, J., and Villadoro, G. JHEP 01, 034 (2016).

[7] Borsanyi, S. et al. Nature 539(7627), 69-71 (2016). 
[8] Svrcek, P. and Witten, E. JHEP 0606, 051 (2006).

[9] Arvanitaki, A., Dimopoulos, S., Dubovsky, S., Kaloper, N., et al. Phys. Rev. D81, 123530 (2010).

[10] Arias-Aragon, F. and Merlo, L. JHEP 10, 168 (2017).

[11] Calibbi, L., Goertz, F., Redigolo, D., Ziegler, R., and Zupan, J. Phys. Rev. D95(9), 095009 (2017).

[12] Di Luzio, L., Mescia, F., Nardi, E., Panci, P., and Ziegler, R. Phys. Rev. Lett. 120(26), 261803 (2018).

[13] Jaeckel, J. and Ringwald, A. Ann. Rev. Nucl. Part. Sci. 60, 405-437 (2010).

[14] Rybka, G. Phys.Dark Univ. 4, 14-16 (2014).

[15] Marsh, D. J. E. Phys. Rept. 643, 1-79 (2016).

[16] Irastorza, I. G. and Redondo, J. Prog. Part. Nucl. Phys. 102, 89-159 (2018).

[17] Tegmark, M., Aguirre, A., Rees, M., and Wilczek, F. Phys. Rev. D73, 023505 (2006).

[18] Kibble, T. W. B. Phys. Rept. 67, 183 (1980).

[19] Gorghetto, M., Hardy, E., and Villadoro, G. JHEP 07, 151 (2018).

[20] Klaer, V. B. and Moore, G. D. JCAP 1710, 043 (2017).

[21] Klaer, V. B. and Moore, G. D. JCAP 1711(11), 049 (2017).

[22] Hiramatsu, T., Kawasaki, M., Saikawa, K., and Sekiguchi, T. Phys.Rev. D85, 105020 (2012).

[23] Ballesteros, G., Redondo, J., Ringwald, A., and Tamarit, C. JCAP 1708(08), 001 (2017).

[24] Sikivie, P. Phys. Rev. Lett. 48, 1156-1159 (1982).

[25] Ringwald, A. and Saikawa, K. Phys. Rev. D93(8), 085031 (2016). Phys. Rev.D94 (4) 049908 (2016).

[26] Visinelli, L. and Gondolo, P. Phys.Rev. D81, 063508 (2010).

[27] Piazza, F. and Pospelov, M. Phys. Rev. D82, 043533 (2010).

[28] Arias, P., Cadamuro, D., Goodsell, M., Jaeckel, J., Redondo, J., et al. JCAP 1206, 013 (2012).

[29] Hogan, C. and Rees, M. Phys.Lett. B205, 228-230 (1988).

[30] Kolb, E. W. and Tkachev, I. I. Phys.Rev.Lett. 71, 3051-3054 (1993).

[31] Kolb, E. W. and Tkachev, I. I. Astrophys.J. 460, L25-L28 (1996).

[32] Vaquero, A., Redondo, J., and Stadler, J. arXiv:1809.09241 [astro-ph.CO] (2018).

[33] Hardy, E. JHEP 02, 046 (2017).

[34] Niikura, H., Takada, M., Yasuda, N., Lupton, R. H., Sumi, T., More, S., More, A., et al. (2017).

[35] Fairbairn, M., Marsh, D. J. E., and Quevillon, J. Phys. Rev. Lett. 119(2), 021101 (2017).

[36] Fairbairn, M., Marsh, D. J. E., Quevillon, J., and Rozier, S. Phys. Rev. D97(8), 083502 (2018).

[37] Inomata, K., Kawasaki, M., Mukaida, K., and Yanagida, T. T. Phys. Rev. D97(4), 043514 (2018).

[38] Katz, A., Kopp, J., Sibiryakov, S., and Xue, W. JCAP 1812, 005 (2018).

[39] Visinelli, L., Baum, S., Redondo, J., Freese, K., and Wilczek, F. Phys. Lett. B777, 64-72 (2018).

[40] Veltmaat, J., Niemeyer, J. C., and Schwabe, B. Phys. Rev. D98(4), 043509 (2018).

[41] Levkov, D. G., Panin, A. G., and Tkachev, I. I. Phys. Rev. Lett. 121(15), 151301 (2018). 
[42] Levkov, D. G., Panin, A. G., and Tkachev, I. I. Phys. Rev. Lett. 118(1), 011301 (2017).

[43] Tkachev, I. I. Sov. Astron. Lett. 12, 305-308 (1986). [Pisma Astron. Zh.12,726(1986)].

[44] Tkachev, I. I. Phys. Lett. B261, 289-293 (1991).

[45] Tkachev, I. In Proceedings, 11th Patras Workshop on Axions, WIMPs and WISPs (Axion-WIMP 2015): Zaragoza, Spain, June 22-26, 2015, 173-178, (2015).

[46] Hertzberg, M. P. and Schiappacasse, E. D. JCAP 1811(11), 004 (2018).

[47] Linde, A. D. Phys. Lett. 158B, 375-380 (1985).

[48] Seckel, D. and Turner, M. S. Phys.Rev. D32, 3178 (1985).

[49] Ade, P. A. R. et al. Astron. Astrophys. 594, A20 (2016).

[50] Matsumura, T. et al. (2013). [J. Low. Temp. Phys.176,733(2014)].

[51] Folkerts, S., Germani, C., and Redondo, J. Phys.Lett. B728, 532-536 (2014).

[52] Visinelli, L. and Gondolo, P. Phys.Rev.Lett. 113, 011802 (2014).

[53] Arvanitaki, A., Baryakhtar, M., and Huang, X. Phys. Rev. D91(8), 084011 (2015).

[54] Cardoso, V., Dias, Ó. J. C., Hartnett, G. S., Middleton, et al. JCAP 1803(03), 043 (2018).

[55] Budker, D., Graham, P. W., Ledbetter, et al. Phys. Rev. X4(2), 021030 (2014).

[56] Barbieri, R., Braggio, et al. Phys. Dark Univ. 15, 135-141 (2017).

[57] Sikivie, P. Phys.Rev.Lett. 51, 1415 (1983).

[58] Du, N. et al. Phys. Rev. Lett. 120(15), 151301 (2018).

[59] Zhong, L. et al. Phys. Rev. D97(9), 092001 (2018).

[60] Melcón, A. Á. et al. JCAP 1805(05), 040 (2018).

[61] Horns, D., Jaeckel, J., Lindner, A., Lobanov, A., Redondo, J., et al. JCAP 1304, 016 (2013).

[62] Caldwell, A., Dvali, G., Majorovits, B. et al. Phys. Rev. Lett. 118(9), 091801 (2017).

[63] Brun, P. et al. Eur. Phys. J. C79(3), 186 (2019).

[64] Baryakhtar, M., Huang, J., and Lasenby, R. Phys. Rev. D98(3), 035006 (2018).

[65] Kahn, Y., Safdi, B. R., and Thaler, J. Phys. Rev. Lett. 117(14), 141801 (2016).

[66] Silva-Feaver, M. et al. IEEE Trans. Appl. Supercond. 27(4), 1400204 (2017).

[67] Sikivie, P., Sullivan, N., and Tanner, D. Phys. Rev. Lett. 112(13), 131301 (2013).

[68] Spector, A. In Proceedings, 12th Patras Workshop on Axions, WIMPs and WISPs (PATRAS 2016): Jeju Island, South Korea, June 20-24, 2016, 133-136, (2017).

[69] Anastassopoulos, V. et al. Nature Phys. 13, 584-590 (2017).

[70] Armengaud, E., Avignone, F., Betz, M., Brax, P., Brun, P., et al. JINST 9, T05002 (2014).

[71] Arvanitaki, A. and Geraci, A. A. Phys.Rev.Lett. 113, 161801 (2014).

[72] Döbrich, B. In Proceedings, Vulcano 2018 workshop, Frascati Physics Series Vol. 66 (2018) arXiv:1807.10170 [hep-ex] (2018). 